Proceedings of the 2011 Winter Simulation Conference

S. Jain, R.R. Creasey, J. Himmelspach, K.P. White, and M. Fu, eds.

\title{
USE OF IDEF-SIM TO DOCUMENT SIMULATION MODELS
}

\author{
João José de Assis Rangel \\ Instituto Federal Fluminense \\ 273 Dr. Siqueira st, Pq. Dom Bosco \\ Campos dos Goytacazes, RJ, 28030-130, BRAZIL
}

\author{
Alessandro Fernandes Nunes \\ Candido Mendes University \\ 100 Anita Pessanha st, Pq. São Caetano \\ Campos dos Goytacazes, RJ, 28030-335, BRAZIL
}

\begin{abstract}
The technique of conceptual modeling IDEF-SIM uses the syntax of IDEFØ and IDEF3 adapted to the peculiarities of the simulation models. It allows the state variables and the logical elements of a discrete system to be abstracted and ordered with most representativeness. However, it is essential to establish additional measures that enhance the process of collecting and authenticating relevant data. This context has motivated this research, which suggests a model documentary that, when associated with the methodology IDEF-SIM, allows recording the details of a dynamic system in an objective and standardized way. We carried a study on a seaport, allowing verifying that the system was translated with greater speed and detail. In this sense, we concluded that the record out in detail increased efficiency in the generation of knowledge about the particularities of the system, regardless of the complexity of the problem and the computer language adopted.
\end{abstract}

\section{INTRODUCTION}

The system of discrete events can provide difficulties in comprehension due to the complexity of the data. As a result, we can verify the construction of models with reduced reliability and effectiveness. According to Ryan and Heavey (2006), in the development of a simulation project, the adoption of measures that enhance the recording of data abstracted by the analyst echoes in the detailed description of the system and enhances the process of creating of the computational model.

Thus, we should develop the conceptual modeling techniques in order to facilitate the capture, visualization and interpretation of the elements of the system. This measure allows for the orderly progression of the study. We will investigate and represent through schemes, pseudo codes and/or verbal descriptions (Maria 1997; Banks et al. 2009; Carson 2004; Pidd 2004; Sargent 2008; Zachman 1987) all functions and variables available in the real environment, as well as the interconnections and rules. We note that we can employ various methodologies, but the best techniques reduce the efforts with the development of the model by rationalizing and integrating all components of the system.

The technique of conceptual modeling IDEF-SIM, proposed by Montevechi et al. (2010), allows the representation of the system in a satisfactory way and with simplified reading. It also offers logical elements for specific projects in the simulation. However, in complex environments, just as with IDEFØ and IDEF3 techniques, only the labeling of the elements and the use of a descriptive framework will not ensure that the model conveys the desired communication. One who knows the technique IDEF-SIM deeply but has little knowledge about the modeled system may not understand the meanings of the information described in the diagram. In this sense, the IDEF-SIM will maximize its efficiency when combined with a documental technique that, in standardized form, may collect and record the specifications of the system. We can list these specifications as the context of the problem and goals that must be met with the design of simulation, the detailed description of the facts and restrictions that constitute each element, and details of the input data. 


\section{Rangel and Nunes}

Through the above context, this paper aims to present a proposal for standardized documentation that supports the conceptual modeling technique IDEF-SIM, increasing the systematic recording of information and data abstracted by the modeler during the development of simulation project.

\section{IDEF-SIM MODELING}

According to Whitman, Huff, and Presley (1997), the use of IDEFØ and IDEF3 techniques allows improvement in the capture and description of information concerning the system, in order to develop the conceptual model in simulation projects. However, the representations of the state variables and of the logic elements available in these techniques, when used separately, may not be sufficient to meet the needs of a simulation project. Simulation projects need grouping and adjusting of the conventional symbols. In this sense, Montevechi et al. (2010) propose a methodology for conceptual modeling called IDEF-SIM that by using the syntax and semantics of IDEF $\varnothing$ and IDEF3 adapted to the peculiarities of the simulation shows to be efficient in the representation of the simulated systems.

\section{METHODOLOGY FOR DOCUMENTATION OF THE CONCEPTUAL MODEL}

In the documentation of models, we must first determine the purpose of the project. We must emphasize the goals to achieve by declaring needs and defining the issues to be answered (Mayer et al. 1995). The question and the response of the real reasons for the problem analysis and the identification of the outcome favor the selection of performance measures and assist in developing the action plan (Carson 2004, Freitas Filho 2008, Ramirez 1998).

According to Mayer et al. (1995), five inquiries must be met for efficient collection and organization of data, namely:

a) Collect the data: This is the detailed log of the observations made in the sequencing of a production process. In this respect, the modeler must agree with the system experts and can capture an initial set of data from the explanation of the process. An efficient way to perform this topic is the previous preparation of the interview, namely: Establish goals and the types of information required; request authorization and scheduling of the interview; and submit, in advance, questions that can stimulate discussion and capture information with higher quality. In order to generate additional information, these interviews may happen throughout the project.

b) classify the data: it lists the productive phases with other elements available on the techniques of process modeling, such as the roles and relationships, the units of behavior, the segments or links, joints, objects, states of the objects and others;

c) organize the information: in this step, the data collected and classified will be sketched in accordance with the syntax of the diagram.

d) approve the scheme: a conceptual model validation is accomplished through following approaches: syntactic analysis and semantic analysis. Still in the semantic analysis, it is verified that the diagram represents the system under study based on information provided by customers and experts involved with the project.

e) refine the layout: the models can be constantly reviewed, so that possible adjustments are made. These refinements will occur by reason of adjustment of information, simplification of the scheme or assignment to elements.

Based on the context above, this paper presents a set of documents intended to assist the registration and interpretation of information available in systems formed by discrete events. Therefore, we pooled and adjusted the IDEF3 Description Summary Form, Standard Diagram Form, IDEF Kit Contents Form, Units of Behavior (UOB) Elaboration Form, Junction Elaboration Form and Object Elaboration Form, 


\section{Rangel and Nunes}

constituents of the IDEFØ and IDEF3 techniques, to the principles and requirements of the modeling technique IDEF-SIM.

Due to the interactivities and characteristics in the generation of knowledge, the documental model suggested for the capture and transcription of the system parameters is not intended to set up a single and sequential approach to record the data. However, we propose the adoption of four forms, adapted to assist the process of examining, collecting, recording and reporting of data, namely: Descriptive Summary IDEF-SIM, Standard Diagram IDEF-SIM, Registration of Legends and Contents IDEF-SIM and Preparation of the Elements in IDEF-SIM. We develop the details of this documental model in Section 4, where we verify its applicability in describing the process of unloading coal at the Ferry Terminal Ponta Ubu - Samarco, located in Espírito Santo, Brazil.

\section{IMPLEMENTING THE PROPOSAL DOCUMENT}

In developing this section, we evaluated the efficiency of the documental model when used as an auxiliary tool in the acquisition of relevant data from the system and especially in supporting the process of computational modeling. To this end, we developed a case study for the system of unloading coal at the Ferry Terminal Ponta Ubu - Samarco - ES, Brazil, which in turn allowed to collect data, record them in the forms proposed, model conceptually the system through the IDEF-SIM and then interpret it in the simulation software Arena ${ }^{\circledR} 12$ (Kelton et al. 2007).

Note that this research does not aim to hold trials for decision making and proposals for improving the ferry terminal, but to submit the documental model to a real environment. We did not consider all the variables present in the unloading of coal but only those that represent the essence of the system. That is in order not to expose the business, and to consider that simplifying the process would not affect the purpose of the study. As for methodology, we collected data through interviews with the technicians responsible for the operations of the port and by direct observation of the system under study.

\subsection{Case Study}

Samarco Mining, founded in 1977, now owned by companies Vale (50\%) and BHP Billiton (50\%), is a producer of iron ore, with a characteristic single production process. This process begins with the extraction of minerals in mining of open pit, followed by improvement in the Concentration Plant. The ore, once concentrated, is carried $396 \mathrm{~km}$ via pipeline to the Pellet Plants at Ponta Ubu, municipality of Anchieta (ES - Brazil), where it is thickened, processed (pellet-feed, sinter feed - or pellets), stored and shipped via a marine terminal (Samarco Mineração SA, 2009).

The Ferry Terminal Ponta Ubu, apart from exporting the production of ore and pellets, is also used in the reception of various charges for their own company and third parties. Among the main raw materials

received, we highlight coal due to the volume moved and the relevance to the impact on the operations of the port.

\subsection{Summary of Unloading Cycle}

The process of unloading, where the coal is removed from the port and transported to the storage area of the company, begins with the early recruitment of dump trucks, which should be available in the ferry terminal before the arrival of the ship. These trucks wait in the waiting area until called to take up their positions in the loading area. In general, the unloading cycle is performed in a sequence (Figure 1). Firstly, the trucks occupy the loading area. Secondly, the load is removed from the ship and placed in trucks with the use of cranes on board and of the grabs. The loaded trucks will only be dispatched to the area of stock when the outflow is available. Once allowed to leave, the coal will be transported to the storage area, and the empty trucks return to the waiting area at the Ferry Terminal, repeating this cycle until the ship is fully unloaded. 


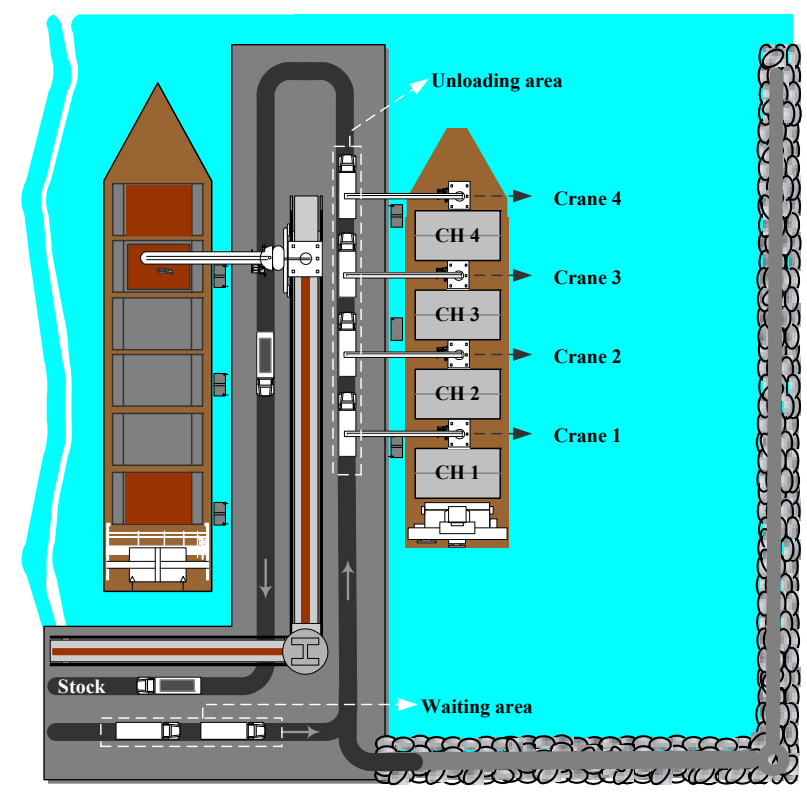

Figure 1: Unloading flow of coal

The lack of physical space for independent maneuvers generates the arrival of trucks in the series, i.e., as the mobile carrier ship (ship loader) occupies the central pier, the only entrance and exit restricts the movement of trucks. It stands out, too, that the combination of the characteristics and peculiarities of the ship employed may be increasing the problems of unloading. In this sense, taking into account the normal operation of the Ferry Terminal Ponta Ubu, the model considered a ship with four cranes and four bays, which, in turn, will order four dump trucks.

\subsection{Formulation of the Documental Model: Collection of Macro Information and Conceptual Modeling}

We relate the development of this step to the use of the forms Descriptive Summary and Standard Diagram. The Descriptive Summary, as shown in Figure 2, is used not only to contextualize the problem and record the goals that must be met with the project, but also to describe the appropriate scenarios to be modeled.

Mayer et al. (1995) describe that, commonly, an initial program of research may vary due to different needs that arise during the project. Therefore, in the Descriptive Summary form, we see recorded information related to the purpose and context of the study, serving as guidelines for analysts not to deviate from the preset goals. The contextualization of the problem should be concise, however, without losing efficiency in the generation of knowledge. In other words, the description should allow a person, unconnected with the case, to understand the process or at least to understand its nature. In the latter case, it will be easier to get the achievement of pending information. Note, also, that the field goal was hypothetically filled by considering that in this work there is not a problem to be solved.

Once collected, the macro information on the system should be outlined in the Standard Diagram form. In this sense, Figure 3 shows the conceptual framework based on IDEF-SIM technique. It refers to the current study (001/09), where the contextual elements were chronologically specified. 


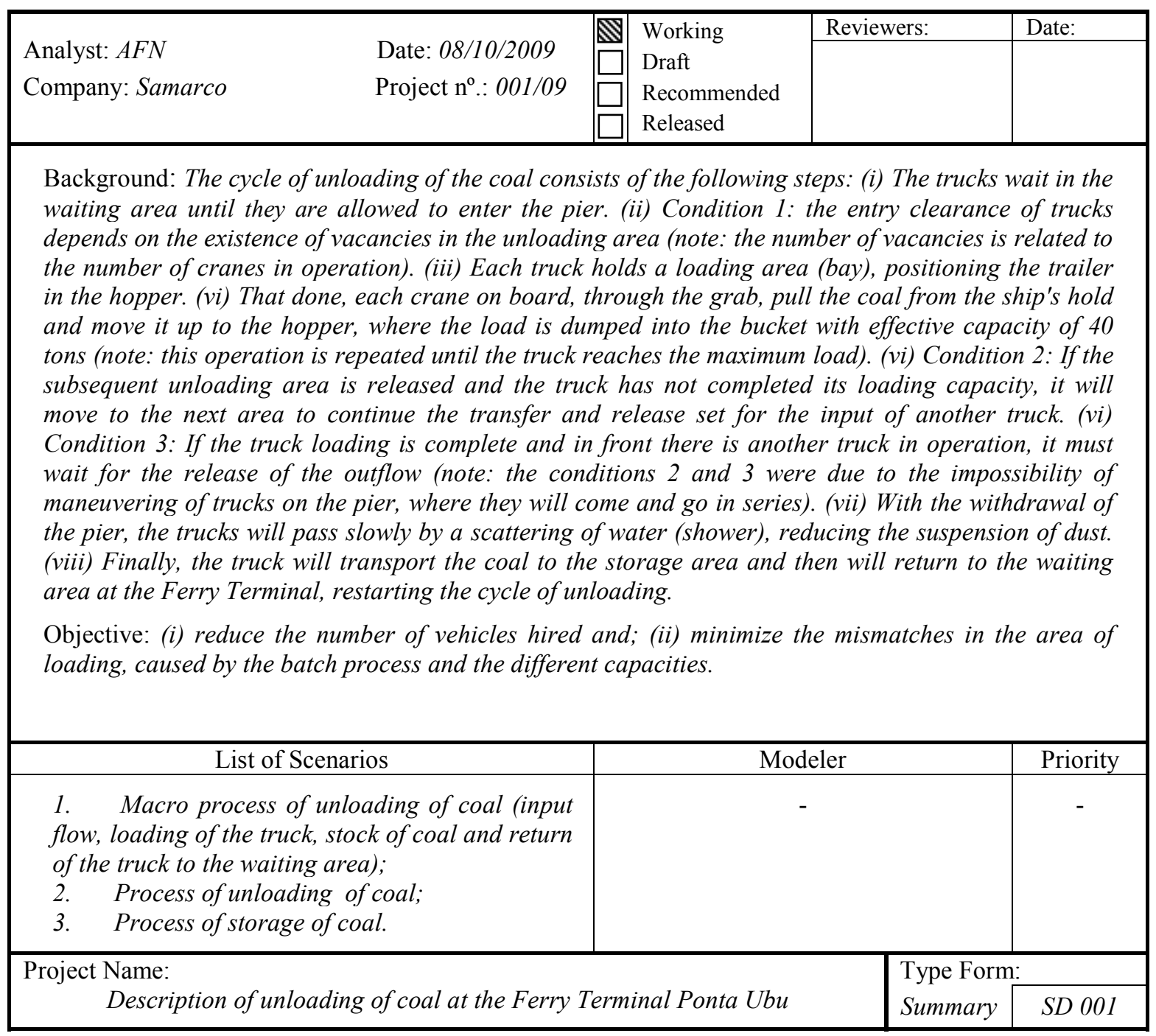

Figure 2: Descriptive Summary of the process of unloading of the coal

The data that composes the documents above is interpreted in different ways. So, for the Descriptive Summary Form, it is respectively reported the name of the analyst responsible for simulation project, the date on which the document was created, the name of the company studied, the number of the project, the project name and the form code. For purposes of planning, there is a list of possible scenarios for the project, as well as it includes the name of those responsible for analyzing and describing the scenarios and the order of priority of this study. The Standard Diagram Form includes the name of the author responsible for developing the conceptual model, the creation date of the document, the name of the project in accordance with the above in the Descriptive Summary. It also includes the reference node for the appointment of the level of decomposition of the scheme and identification of which element, of the parent diagram, is being detailed. The title and the code / page of the document close this Form. Finally, for both forms, the conditions of the model, the names of the reviewers and the date of occurrence of reviews are described. Under the conditions of the model, it is specified the situation where the document is. Thus, in the Working item there is an indication that the scheme analyzed is under construction or that there is a significant change being developed, regardless of previous status. The Draft states that one needs to make minor corrections to the previous scheme before accepting it. That is based on the opinions 


\section{Rangel and Nunes}

of a number of analysts. When the Recommended field is registered, it is understood that the document is providing information regarding the review and approval of the project, not being used for future changes. In the Released field, it is informed that the document is in the final version and may be published.

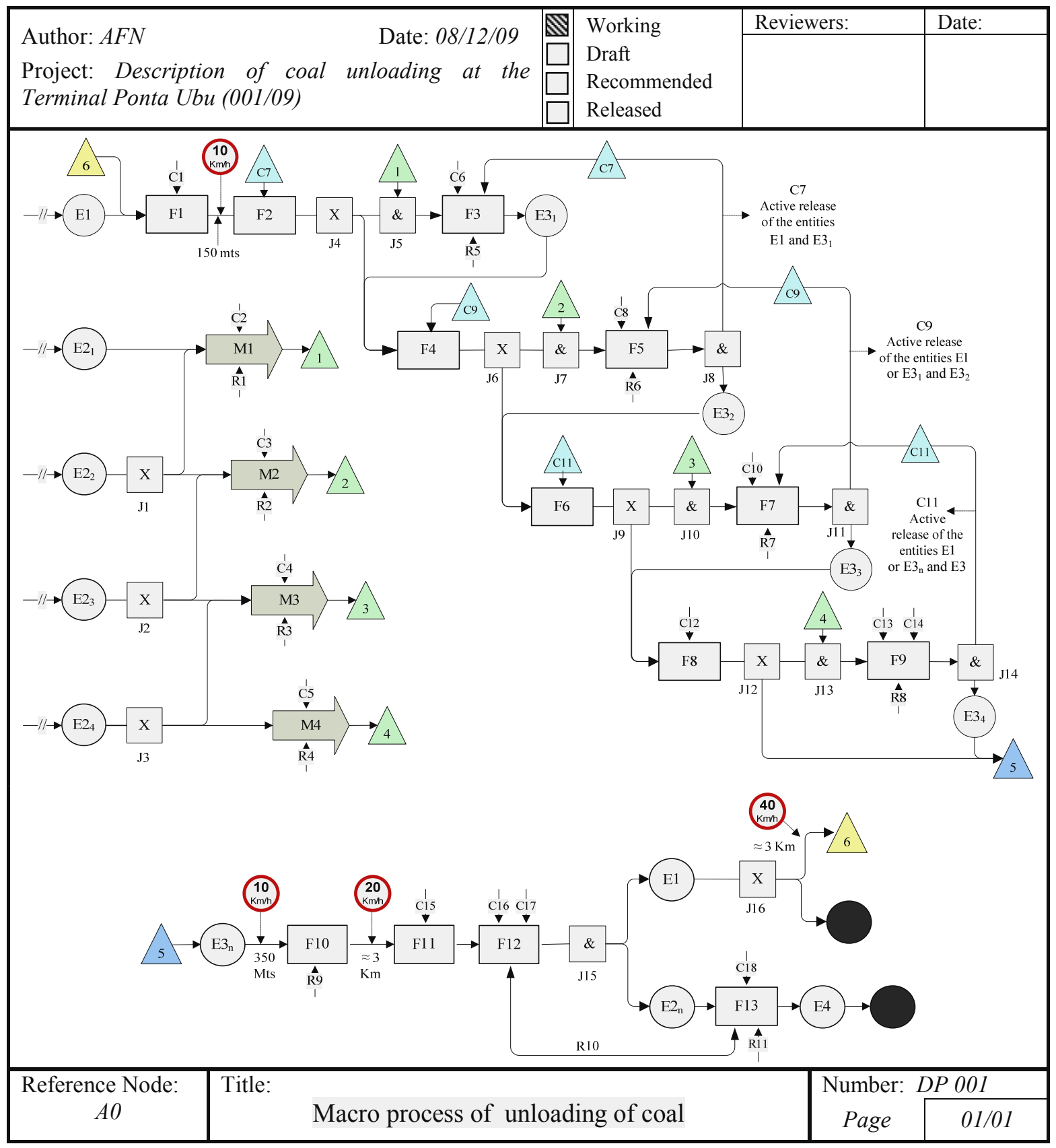

Figure 3: Standard Diagram of the macro process of unloading of coal

\subsection{Formulation of Documental Model: Collection, Treatment and Data Registration}

The main elements of IDEF-SIM technique used to lay out the system (Standard Diagram) should be labeled, in a concise way, in the Contents and Legends Registration form. The description of the elements in this form (type, code and title / description) could be developed in a sequential manner, respecting the 
order of their occurrence in the diagram and / or group of elements. In order to facilitate understanding, the use of this tool is explained in Figure 4.

\begin{tabular}{|c|c|c|c|c|c|}
\hline \multicolumn{3}{|c|}{$\begin{array}{l}\text { Author: } A F N \quad \text { Date: } 08 / 12 / 09 \\
\text { Project: Description of unloading of coal at Ferry Terminal Ponta } \\
\qquad \text { Ubu (001/09) }\end{array}$} & $\begin{array}{l}\text { Working } \\
\text { Draft } \\
\text { Recommended } \\
\text { Released }\end{array}$ & \multicolumn{2}{|c|}{$\begin{array}{l}\square \text { Standard } \\
\square \text { Update }\end{array}$} \\
\hline Type & Code & \multicolumn{3}{|l|}{ Title / Description } & Page \\
\hline 1 & $E 1$ & \multicolumn{3}{|l|}{ Empty truck } & 01 \\
\hline 1 & $E 2_{1}$ & \multicolumn{3}{|l|}{ Coal available in the hold 1 of the ship } & 01 \\
\hline 1 & $E 2_{2}$ & \multicolumn{3}{|l|}{ Coal available in the hold 2 of the ship } & 01 \\
\hline 1 & $E 2_{3}$ & \multicolumn{3}{|l|}{ Coal available in the hold 3 of the ship } & 01 \\
\hline 1 & $E 2_{4}$ & \multicolumn{3}{|l|}{ Coal available in the hold 4 of the ship } & 01 \\
\hline 1 & $E 3_{1}$ & \multicolumn{3}{|l|}{ Truck loaded in bay 1} & 01 \\
\hline 1 & $E 3_{2}$ & \multicolumn{3}{|l|}{ Truck loaded in bay 2} & 01 \\
\hline 1 & $E 3_{3}$ & \multicolumn{3}{|l|}{ Truck loaded in bay 3} & 01 \\
\hline 1 & $E 3_{4}$ & \multicolumn{3}{|l|}{ Truck loaded in bay 4} & 01 \\
\hline 1 & $E 4$ & \multicolumn{3}{|l|}{ Coal stacked in the storage area } & 01 \\
\hline 3 & M1 & \multicolumn{3}{|c|}{ Getting the coal from the hold of the ship into bay 1} & 01 \\
\hline 5 & $C 2$ & \multicolumn{3}{|c|}{ Check if the truck is positioned in the bay or is already loaded } & 01 \\
\hline 6 & $R 1$ & \multicolumn{3}{|c|}{ Crane board 1, grab 1 and crane operator 1 (docker) } & 01 \\
\hline 3 & M2 & \multicolumn{3}{|c|}{ Getting the coal from the hold of the ship into bay 2} & 01 \\
\hline 5 & C3 & \multicolumn{3}{|c|}{ Check if the truck is positioned in the bay or is already loaded } & 01 \\
\hline 6 & $R 2$ & \multicolumn{3}{|c|}{ Crane board 2, grab 2 and crane operator 2 (docker) } & 01 \\
\hline \multicolumn{4}{|c|}{ Title: Macro process of unloading of coal } & \multicolumn{2}{|c|}{ Ref. Node: $A O$} \\
\hline \multicolumn{4}{|c|}{$\begin{array}{l}\text { Element Type: } 1 \text {-Entities; } 2 \text {-Functions; } 3 \text {-Drive; } 4 \text {-Rules; } 5 \text {-Control; } 6- \\
\text { Means; } 7 \text {-Others }\end{array}$} & Page & 01 \\
\hline
\end{tabular}

Figure 4: Registration of Legends and Contents of the macro process of unloading of coal

Considering that the objective of this research is to present the applicability of the documental draft and that the conceptual model IDEF-SIM, which refers to the Macro process of Unloading of Coal, is composed of 76 elements highlighted, only 16 of these elements were labeled in the form above, being sufficient to demonstrate the form and the simple process to fill the form.

The Contents and Legends Registration form aims to facilitate the reading and interpretation of the Standard Diagram. However, as this is a brief description, some information of the system such as the objects involved (entities), the summary of the facts, the case constraints (restrictions) and the design of the input data may be omitted or constructed without clarity, especially in environments with higher levels of complexity. In this sense, to increase efficiency in detailing the features and characteristics of the elements of a system, its characteristics should be recorded in the form Preparation of the Elements. This measure allows the transcription of pertinent information that would hardly be expressed in the schematic model and simplifies the process of review and validation of the conceptual model IDEF-SIM.

In this section, to demonstrate the functionality of the form Preparation of the Elements (Figures 5 and 6), only the elements E1 (empty truck) and M1 (Getting the coal from the hold of the ship to the truck located in bay 1) have been elucidated. 


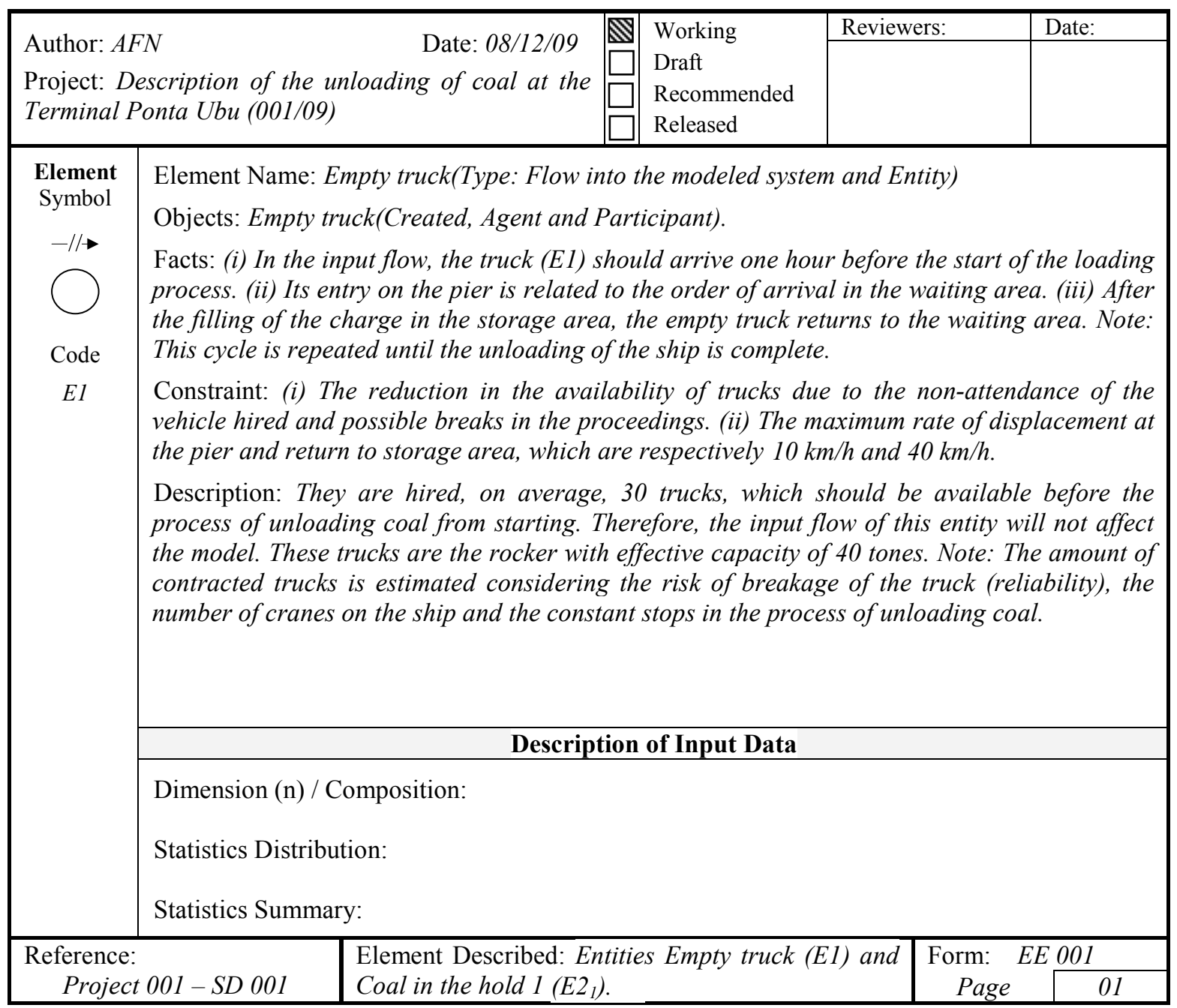

Figure 5: Form for the Preparation of Elements of the conceptual model, element E1

It is observed that the data of the headers that compose the Contents and Legends Registration and Preparation of Elements are identical to the Standard Diagram form, keeping the same meaning. However, other fields are interpreted as follows: For the Contents and Legends Registration form in Figure 4, the kit Standard reports that the model was developed in an orderly manner and the kit Update is adopted to record the final version and to indicate that updated information were added to an existing and validated model. In the body of the document, they are informed the type of element, the element tag in accordance with the conceptual model, a brief description of the element and Standard Diagram page in which the element has been described. Finally, at the bottom, it is described the title of the system under study, the reference number of the element type (coded 1 to 7 ), the reference node of the diagram documented and pagination of the form. In the Preparation of the Elements form in Figure 5, one can see that fields are suitable for all elements of the methodology IDEF-SIM, avoiding the use of various documents. In this sense, in the Element Name field, it is briefly described the functionality of the element in the modeled system. The Objects represent the physical or informational entities that interact directly with the element documented, and can be created, modified or destroyed in the process. Mayer et al. (1995) add that the objects of interest can be classified as Agent (when the object to be subjected to a processing unit plays an active role), Affected ((when the object receives the processing by a unit), 


\section{Rangel and Nunes}

Participant (when the object does not suffer or cause changes when interacting with the processing unit) and Created or Destroyed (when the object, respectively, is created or destroyed by a processing unit). In the field Facts, the main actions and measures are in context, giving meaning to the events represented by the elements under study. The Constraint is a field created to list the restrictions or obligations on the system modeled. In the Description, one can detail the characteristics of each element, such as its function, interactions (links input and output), the resources used and the controls established. When the interaction between the static and dynamic elements of a system is time consuming, either to insertion or systematic processing of a temporary entity, the statistics of Input Data needs to be documented. Due to the learning curve and changes in the process, this information needs to be constantly reassessed.

In summary, the proposed forms for the documentation of discrete event intend to collect, sort and organize data, but also to facilitate the process of verifying and refining the schema. In this sense, it seeks, efficiently, to represent the way the system operation takes place and not the way it will be developed in simulation software. This move enables an analyst, experienced or not, to get the information needed to develop the computational model, regardless of language used.

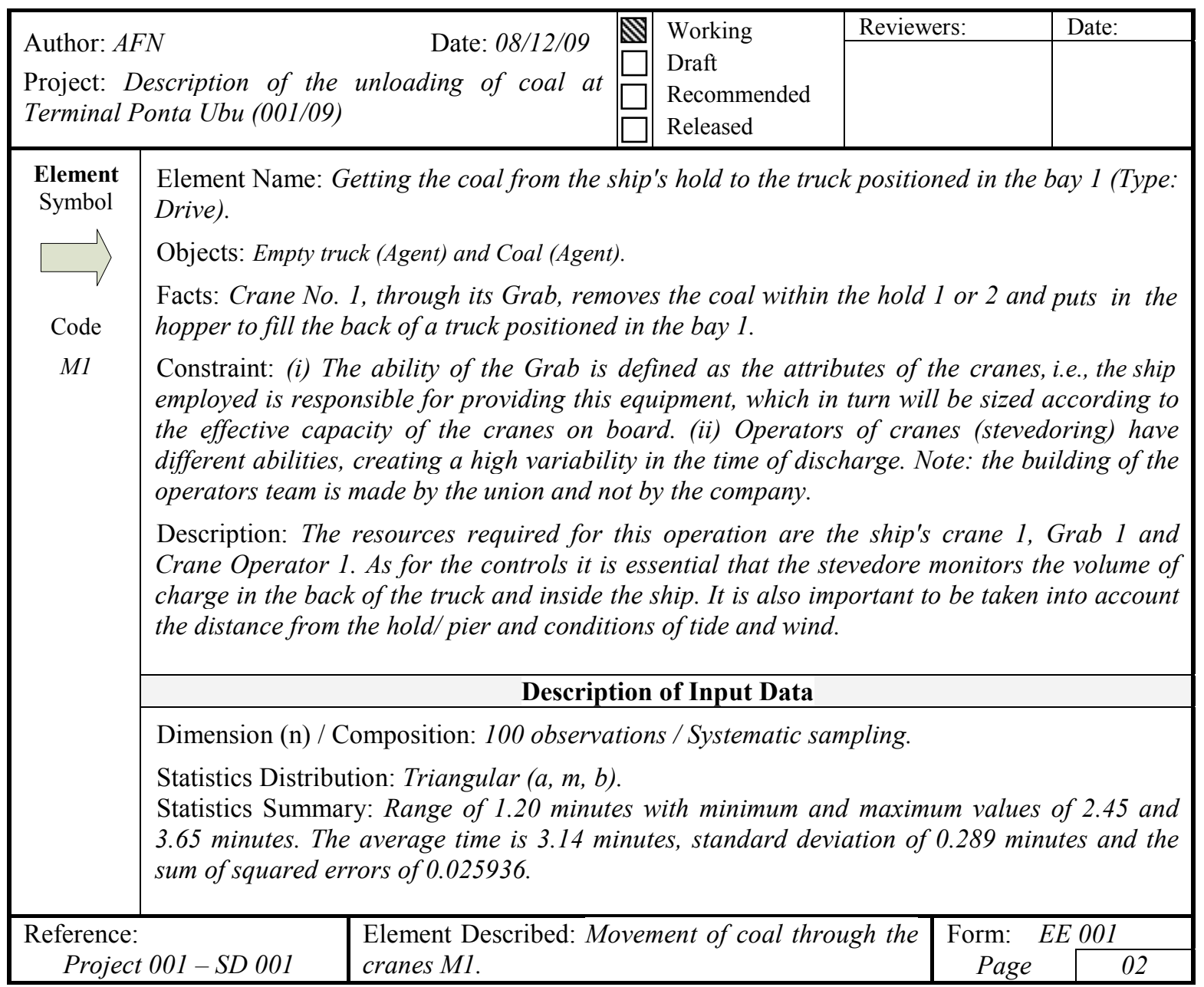

Figure 6: Form for the Preparation of Elements of the conceptual model, element M1 


\section{TRANSLATION OF THE DOCUMENTAL MODEL IN ARENA ${ }^{\circledR} 12$}

The translation efficiency of the conceptual model requires that the main events of the system are reported clearly. In this sense, the development of a computational model (Figure 7) relied on information in the Descriptive Summary, Contents and Legends Registration and Preparation of the Elements forms.

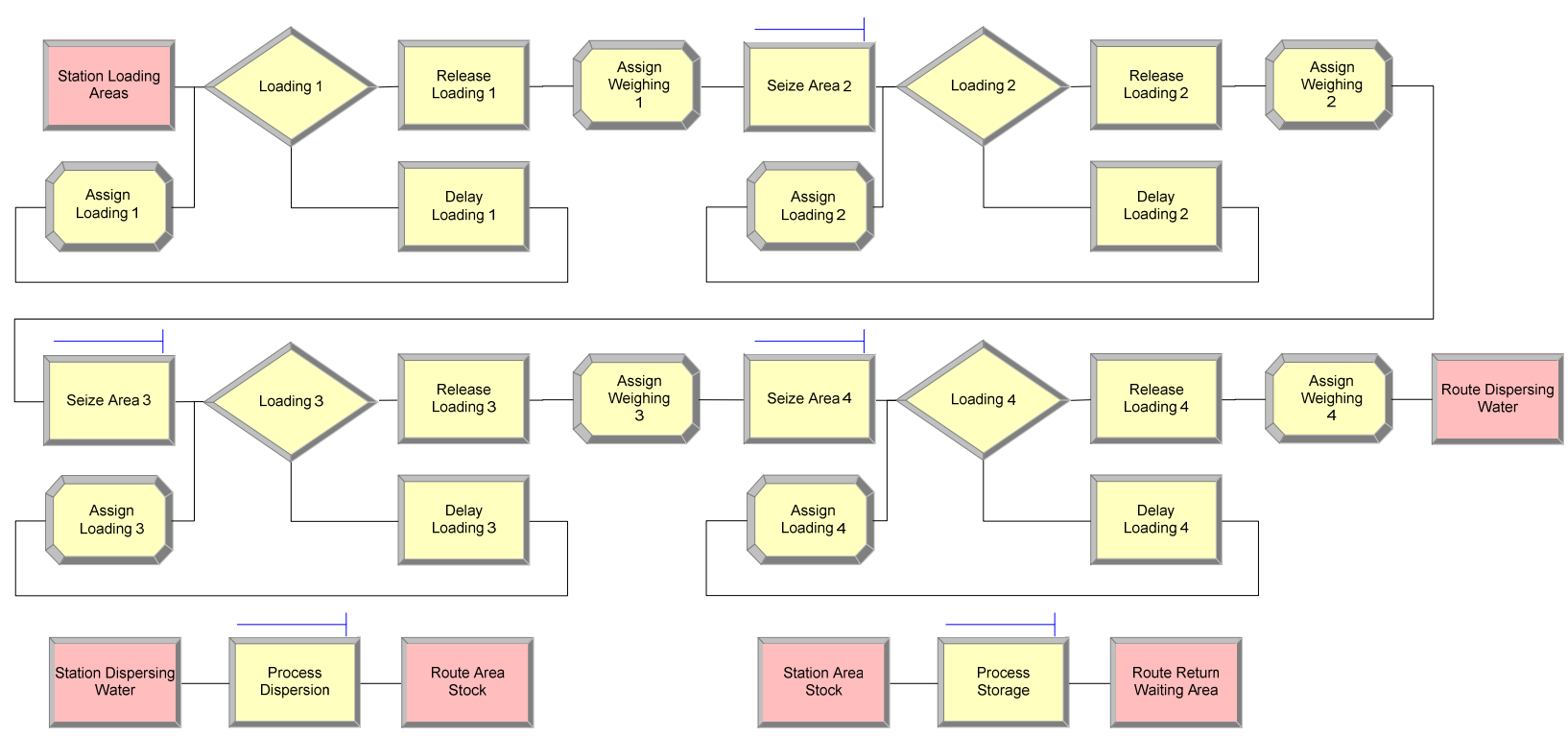

Figure 7: Computational modeling (Arena $\left.{ }^{\circledR} 12\right)$ of the system of unloading of coal

The modules Assign and Decide establish the logical parameters responsible for monitoring the dynamics of the model. Initially, there is an Assign module that adds an attribute, named Charge, to each entity that enters the system modeled. This attribute, then, is used to control the amount of charge added to the truck and allows that the Decide modules, through its logic control, occupy the loading bays. The occupation is made in decreasing order, starting from the last (Bay 4) for the first (Bay 1). The release control of the bays is also run by the Decide module, where the truck will be released, when the subsequent bay is available and the Loading variable $n$ reaches the preset value. The combination of the modules Station as a destination and Route as a source enables that the time consumed with the displacement is adequately represented in the model.

\section{WHAT HAVE WE LEARNED?}

In developing this model, it was evident that the adoption of the proposal document increased efficiency in the generation of knowledge and thus facilitated the construction and verification of the computational model. In complex environments, just as with IDEFØ and IDEF3 techniques, only the labeling of the elements and the use of a descriptive framework will not ensure that the model conveys the required communication. A person, who deeply knows the technique IDEF-SIM, but has little knowledge about the modeled system, may not understand the meanings of the information described in the diagram. In this sense, the IDEF-SIM will have maximized its efficiency when combined with a documental technique that, in standardized form, may collect and record the specifics of the system. 


\section{ACKNOWLEDGMENTS}

The authors would like to thank the National Council for Scientific and Technological Development CNPq, and the Foundation for Research Support of Rio de Janeiro - FAPERJ for financial support for this research.

\section{REFERENCES}

Banks, J., J. S. Carson, B. L. Nelson, and D. M. Nicol. 2009. Discrete-Event System Simulation. 5th ed. New Jersey: Prentice-Hall, Inc.

Carson, J. S. 2004. "Introduction to Modeling and Simulation." In Proceedings of the 2004 Winter Simulation Conference, edited by R .G. Ingalls, M. D. Rossetti, J. S. Smith, and B. A. Peters, 9-16. Piscataway, New Jersey: Institute of Electrical and Electronics Engineers, Inc.

Freitas Filho, P. J. 2008. Introdução à Modelagem e Simulação de Sistemas: com Aplicações em Arena. Second edition. Florianópolis: Visual Books, Inc - Brazil.

Kelton, D. W., R. P. Sadowski, and D. T. Sturrock. 2007. Simulation with Arena. 4th ed. New York: McGraw Hill.

Maria, A. 1997. "Introduction to Modeling and Simulation." In Proceedings of the 1997 Winter Simulation Conference, edited by S. Andradóttir, K. J. Healy, D. H. Withers, and B. L. Nelson, 7-13. Piscataway, New Jersey: Institute of Electrical and Electronics Engineers, Inc.

Mayer, R. J., C. P. Menzel, M. K. Painter, P. S. Witte, T. Blinn and B. Perakath. 1995. Information Integration for Concurrent Engineering (IICE) idef3 process description capture method report. Technical report, Knowledge Based Systems Inc., University Drive East, College Station, Texas.

Montevechi, J. A. B., F. Leal, A. F. Pinho, R. F. da S. Costa, M. L. M. de Oliveira, and A. L. F. da Silva. 2010. "Conceptual Modeling in Simulation Projects by Mean Adapted IDEF: An Application in a Brazilian Tech Company." In Proceedings of the 2010 Winter Simulation Conference, edited by B. Johansson, S. Jain, J. Montoya-Torres, J. Hugan, and E. Yücesan, 1624-1635. Piscataway, New Jersey: Institute of Electrical and Electronic Engineers, Inc.

Pidd, M. 2004. Computer Simulation in Management Science. 5th ed. Chichester: John Wiley \& Sons Ltd., Inc.

Ramirez, W. F. 1998. Computational Methods in Process Simulation. Second edition. Boston: Butterworth-Heinemann, Inc.

Ryan, J. and C. Heavey. 2006. "Process Modeling for Simulation." Computers in Industry. Elsevier 57: 437-450.

Samarco Mineração S.A. 2005. Annual Report Samarco. Accessed November 10, 2009. http://www.samarco.com.

Sargent, R. G. 2008. "Verification and Validation of Simulation Models." In Proceedings of the 2008 Winter Simulation Conference, edited by S. J. Mason, R. R. Hill, L. Mönch, O. Rose, T. Jefferson, and J. W. Fowler, 157-169. Piscataway, New Jersey: Institute of Electrical and Electrical Engineers, Inc.

Whitman, L., B. Huff and A. Presley. 1997. "Structured Models and Dynamic Systems Analysis: the Integration of the IDEF0/IDEF3 Modeling Methods and Discrete Event Simulation." In Proceedings of the 1997 Winter Simulation Conference, edited by S. Andradóttir, K. J. Healy, D. H. Withers, and B. L. Nelson, 518-524. Piscataway, New Jersey: Institute of Electrical and Electronics Engineers, Inc.

Zachman, J. A. 1987. “A Framework for Information Systems Architecture." IBM Systems Journal 26(3):276-292. 


\section{AUTHOR BIOGRAPHIES}

JOÃO JOSÉ DE ASSIS RANGEL is a professor at Federal Fluminense Institute (IFF) and Candido Mendes University (UCAM) in Campos dos Goytacazes, RJ - Brazil. He received a doctoral degree in Materials Engineering at UENF in 1998. His research interests include simulation on logistics and manufacturing, simulation on super hard materials process and simulation education. His e-mail address is joao@ucam-campos.br.

ALESSANDRO FERNANDES NUNES is an economist and MSc in Operations Research and Computational Intelligence at Candido Mendes University (UCAM). His e-mail address is alessandrofnunes@gmail.com. 